\title{
Clinical presentations as predictors of prolonged mechanical ventilation in Guillain-Barré syndrome in an institution with limited medical resources
}

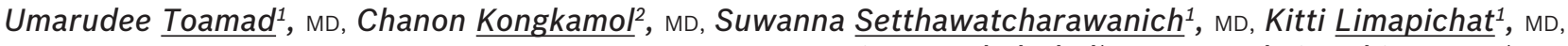
Kanitpong Phabphal $^{1}$, MD, Pornchai Sathirapanya ${ }^{1}$, MD

\begin{abstract}
INTRODUCTION Severe Guillain-Barré syndrome (GBS) causes ventilatory insufficiency and the need for prolonged artificial ventilation. Under circumstances where medical care for patients with severe GBS is required in a resourcelimited institution, identifying initial clinical presentations in GBS patients that can predict respiratory insufficiency and the need for prolonged mechanical ventilation (> 15 days) may be helpful for advanced care planning.

METHODS The medical records of patients diagnosed with GBS in a tertiary care and medical teaching hospital from January 2001 to December 2010 were retrospectively reviewed. The demographic data and clinical presentations of the patients were summarised using descriptive statistics. Clinical predictors of respiratory insufficiency and the need for prolonged mechanical ventilation (> 15 days) were identified using univariate logistic regression analysis.

RESULTS A total of 55 patients with GBS were included in this study. Mechanical ventilation was needed in 28 ( $50.9 \%$ ) patients. Significant clinical predictors for respiratory insufficiency were bulbar muscle weakness (odds ratio [OR] 5.08, 95\% confidence interval $[\mathrm{Cl}] 1.31-21.60, \mathrm{p}=0.007)$ and time to peak limb weakness $\leq 5$ days (OR $0.75,95 \% \mathrm{Cl} 0.62-0.91$, $p<0.001)$. Bulbar muscle weakness $(p=0.006)$ and time to peak limb weakness $\leq 5$ days $(p<0.001)$ were also found to be significantly associated with the need for prolonged mechanical ventilation ( $>15$ days).

CONCLUSION Bulbar weakness and time to peak limb weakness $\leq 5$ days were able to predict respiratory insufficiency and the need for prolonged mechanical ventilation in patients with GBS.
\end{abstract}

Keywords: Guillain-Barré syndrome, mechanical ventilation, respiratory failure

\section{INTRODUCTION}

Guillain-Barré syndrome (GBS) was first described in 1916. (1) It is a devastating neurological disease. The acute phase of the disease is characterised by generalised paralysis, bulbar muscle weakness and respiratory failure. Immune-mediated inflammation of the myelin sheath of peripheral nerves is a known pathogenesis. ${ }^{(2)}$ The overall incidence of GBS ranges from $1.1: 100,000$ to $1.8: 100,000$, but is higher $(1.7-3.3: 100,000)$ among persons aged above 50 years. ${ }^{(3)}$ Antecedent systemic infections, such as respiratory tract infections and gastroenteritis, are associated with $70 \%$ of all GBS cases. ${ }^{(3)}$ For these patients, complications related to prolonged mechanical ventilation and immobilisation are significant causes of morbidity and mortality. As GBS patients may benefit from early treatment (e.g. tracheostomy and intensive respiratory care), this retrospective study was conducted to determine the clinical presentations of patients with GBS that are associated with the need for: (a) mechanical ventilation; and (b) prolonged mechanical ventilation ( $>15$ days).

\section{METHODS}

Patients aged $\geq 15$ years who were diagnosed with GBS (according to Asbury and Cornblath's diagnostic criteria) ${ }^{(4)}$ in Songklanagarind Hospital, Hat Yai, Thailand, from January
2001 to December 2010 were included in this study. Patients who had other specific types of polyneuropathy (e.g. diabetic polyneuropathy, thiamine deficiency polyneuropathy, polyneuropathy due to industrial agents or drugs, porphyria and polyneuropathy associated with human immunodeficiency virus infection) were excluded. Electrophysiological criteria for the diagnosis of GBS were met in all enrolled cases.

The following data was collected from the medical records of all enrolled patients: patient demographics; history of antecedent infections (e.g. respiratory tract infection and gastroenteritis) or vaccinations; presenting neurological symptoms; Hughes severity score (HSS) at presentation and discharge; time to peak limb weakness; results of cerebrospinal fluid (CSF) analysis; total number of days for which ventilation support was needed; and treatment options. Time to peak limb weakness refers to the duration from the onset of the first neurological symptoms to the time the most severe limb weakness was experienced. Clinical presentations that were predictors of respiratory insufficiency (i.e. the need for ventilator support) and the need for prolonged ventilation support (> 15 days) were determined by univariate logistic regression analysis.

Data was presented as number (percentage), mean \pm standard deviation and/or median (1st quartile [Q1], 3rd quartile [Q3]).

${ }^{1}$ Division of Neurology, Department of Internal Medicine, ${ }^{2}$ Occupational Health Unit, Department of Community Medicine, Faculty of Medicine, Prince of Songkla University, Hat Yai, Songkhla, Thailand

Correspondence: Dr Pornchai Sathirapanya, Associate Professor, Division of Neurology, Department of Internal Medicine, Faculty of Medicine, Prince of Songkla University, Hat Yai, Songkhla 90110, Thailand. sporncha@medicine.psu.ac.th 
Inferential statistics, with a 95\% confidence interval, were found using Mann-Whitney $U$ test, chi-square test, Fisher's exact test and a logistic regression model. A p-value $<0.05$ indicated statistical significance. Statistical analyses were performed using The R Project for Statistical Computing version 2.15.2 (The R Foundation, Vienna, Austria), with the software packages commed version 1.0 and epid version 2.11.0.1.

\section{RESULTS}

A total of 55 patients with GBS who met the inclusion criteria were identified using the hospital's computer information system. Among these 55 patients, 28 (50.9\%) were male and 27 (49.1\%) were female. The mean age was $50.15 \pm 15.84$ (range $17-86)$ years. Antecedent infections were reported in 14 (25.5\%) of the patients; $10(18.2 \%)$ patients had upper respiratory tract infections and $4(7.3 \%)$ had gastroenteritis. Two patients had been vaccinated before the onset of GBS - one with a rabies vaccine and the other with an influenza vaccine. 54 (98.2\%) of the 55 patients presented with progressive weakness in more than one limb. Additionally, 20 (36.4\%) had bulbar muscle weakness, 18 (32.7\%) had lower motor neuron facial paresis (unilateral or bilateral) and $4(7.3 \%)$ had ptosis. The mean time to peak limb weakness was $6.96 \pm 4.90$ (range 1-21) days. The patients' HSS at admission were as follows: score $1(\mathrm{n}=0)$; score $2(n=2,3.6 \%)$; score $3(n=13,23.6 \%)$; score $4(n=23$, $41.8 \%)$; and score $5(n=17,30.9 \%)$. The mean CSF protein level was $178.37 \pm 187.78$ (range $24-980) \mathrm{mg} / \mathrm{dL}$ and the mean CSF white blood cell count was $4.02 \pm 8.59$ (range $0-50$ ) cells $/ \mathrm{mm}^{3}$ (Table I).

Among the 55 patients, 28 (50.9\%) had respiratory failure that required mechanical ventilation. Among these 28 patients, $20(71.4 \%)$ required prolonged mechanical ventilation (> 15 days), 5 (17.9\%) were mechanically ventilated for less than 15 days and $3(10.7 \%)$ were discharged against advice or transferred. A tracheostomy was indicated in 10 of the 28 (35.7\%) patients. The mean number of days on mechanical ventilation was $30.68 \pm 62.05$ (range 6-330) days. All mechanically ventilated patients were treated using plasma exchange or intravenous immunoglobulin.

In this study, we found that bulbar muscle weakness and shorter time to peak limb weakness were significant predictors of the need for mechanical ventilation. There was a significantly higher proportion of patients with bulbar muscle weakness in the group of 28 patients who were mechanically ventilated $(\mathrm{n}=15,53.6 \%)$ than in the group of 27 patients who were not mechanically ventilated ( $\mathrm{n}=5,18.5 \%$ ) (odds ratio [OR] 5.08, $95 \%$ confidence interval $[\mathrm{Cl}] 1.31-21.60, p=0.007)$. The median time to peak limb weakness was significantly shorter among the patients who were mechanically ventilated (5 days; $\mathrm{Q} 1, \mathrm{Q} 3=2.5$, 5.5) than among the patients who were not mechanically ventilated (8 days; Q1, Q3 = 6.0, 13.0) (OR 0.75, 95\% Cl 0.62-0.91, p < 0.001) (Table II). Other factors, such as age, gender, antecedent upper respiratory tract infection, ptosis, facial paresis, level of CSF protein and CSF white blood cell count, were not found to be associated with the need
Table I. Baseline clinical characteristics of the patients with Guillain-Barré syndrome $(n=55)$.

\begin{tabular}{|c|c|}
\hline Characteristic & No. (\%) \\
\hline Age* (yr) $^{*}$ & $50.15 \pm 15.84(17-86)$ \\
\hline \multicolumn{2}{|l|}{ Gender } \\
\hline Male & $28(50.9)$ \\
\hline Female & $27(49.1)$ \\
\hline \multicolumn{2}{|l|}{ Antecedent infection } \\
\hline Upper respiratory tract & $10(18.2)$ \\
\hline Gastroenteritis & $4(7.3)$ \\
\hline \multicolumn{2}{|l|}{ Initial presentation } \\
\hline Limb weakness & $54(98.2)$ \\
\hline Limb numbness & $45(81.8)$ \\
\hline $\begin{array}{l}\text { Lower motor neuron facial paresis } \\
\text { (unilateral or bilateral) }\end{array}$ & $18(32.7)$ \\
\hline Bulbar muscle weakness & $20(36.4)$ \\
\hline Ptosis & $4(7.3)$ \\
\hline Time to peak limb weakness* (day) & $6.96 \pm 4.90(1-21)$ \\
\hline Cerebrospinal fluid protein* (mg/dL) & $178.37 \pm 187.78(24-980)$ \\
\hline 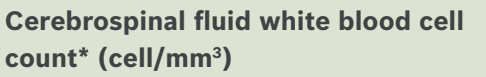 & $4.02 \pm 8.59(0-50)$ \\
\hline
\end{tabular}

*Data presented as mean \pm standard deviation (range).

Table II. Comparison of the baseline clinical characteristics of patients with Guillain-Barré syndrome, according to their need for mechanical ventilation.

\begin{tabular}{|c|c|c|c|}
\hline \multirow[t]{2}{*}{ Characteristic } & \multicolumn{2}{|c|}{ No. (\%) } & \multirow[t]{2}{*}{ p-value } \\
\hline & $\begin{array}{l}\text { Not ventilated } \\
\quad(n=27)\end{array}$ & $\begin{array}{l}\text { Ventilated } \\
(n=28)\end{array}$ & \\
\hline 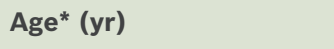 & $48.3 \pm 16.4$ & $51.9 \pm 15.3$ & $0.45^{*}$ \\
\hline Gender & & & $0.50^{\S}$ \\
\hline Male & $12(44.4)$ & $16(57.1)$ & \\
\hline Female & $15(55.6)$ & $12(42.9)$ & \\
\hline \multicolumn{4}{|l|}{ Antecedent infection } \\
\hline Upper respiratory tract & $7(25.9)$ & $3(10.7)$ & $0.27^{\S}$ \\
\hline Gastroenteritis & $1(3.7)$ & $3(10.7)$ & $0.61^{\natural}$ \\
\hline \multicolumn{4}{|l|}{ Initial presentation } \\
\hline Limb weakness & $26(96.3)$ & $28(100.0)$ & 0.49 \\
\hline Limb numbness & $23(85.2)$ & $22(78.6)$ & $0.73^{\natural}$ \\
\hline $\begin{array}{l}\text { Lower motor } \\
\text { neuron facial paresis } \\
\text { (unilateral or bilateral) }\end{array}$ & $10(37.0)$ & $8(28.6)$ & $0.70^{\S}$ \\
\hline Bulbar muscle weakness & $5(18.5)$ & $15(53.6)$ & $0.007^{\S}$ \\
\hline Ptosis & $2(7.4)$ & $2(7.1)$ & $0.73^{\pi}$ \\
\hline $\begin{array}{l}\text { Time to peak limb } \\
\text { weakness }^{+} \text {(day) }\end{array}$ & $8(6.0,13.0)$ & $5(2.5,5.5)$ & $<0.001^{\|, * *}$ \\
\hline $\begin{array}{l}\text { Cerebrospinal fluid } \\
\text { protein }^{+}(\mathrm{mg} / \mathrm{dL})\end{array}$ & $\begin{array}{c}120.6 \\
(63.0,286.9)\end{array}$ & $\begin{array}{c}108 \\
(66.3,157.0)\end{array}$ & $0.80^{\prime \prime}$ \\
\hline
\end{tabular}

*Data presented as mean \pm standard deviation. tData presented as median (quartile ${ }^{1}$, quartile ${ }^{3}$ ). p-value calculated using ${ }^{\ddagger} t$-test; $\S$ chi-square test; ${ }^{\text {I }}$ Fisher's

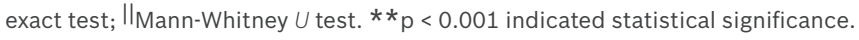

for mechanical ventilation. When the need for prolonged mechanical ventilation (> 15 days) was considered, we found that both bulbar muscle weakness $(p=0.006)$ and time to peak limb weakness $\leq 5$ days $(p<0.001)$ were significant predictors (Table III). 
Table III. Comparison of the baseline clinical characteristics of the patients with Guillain-Barré syndrome, according to whether the patient received mechanical ventilation.

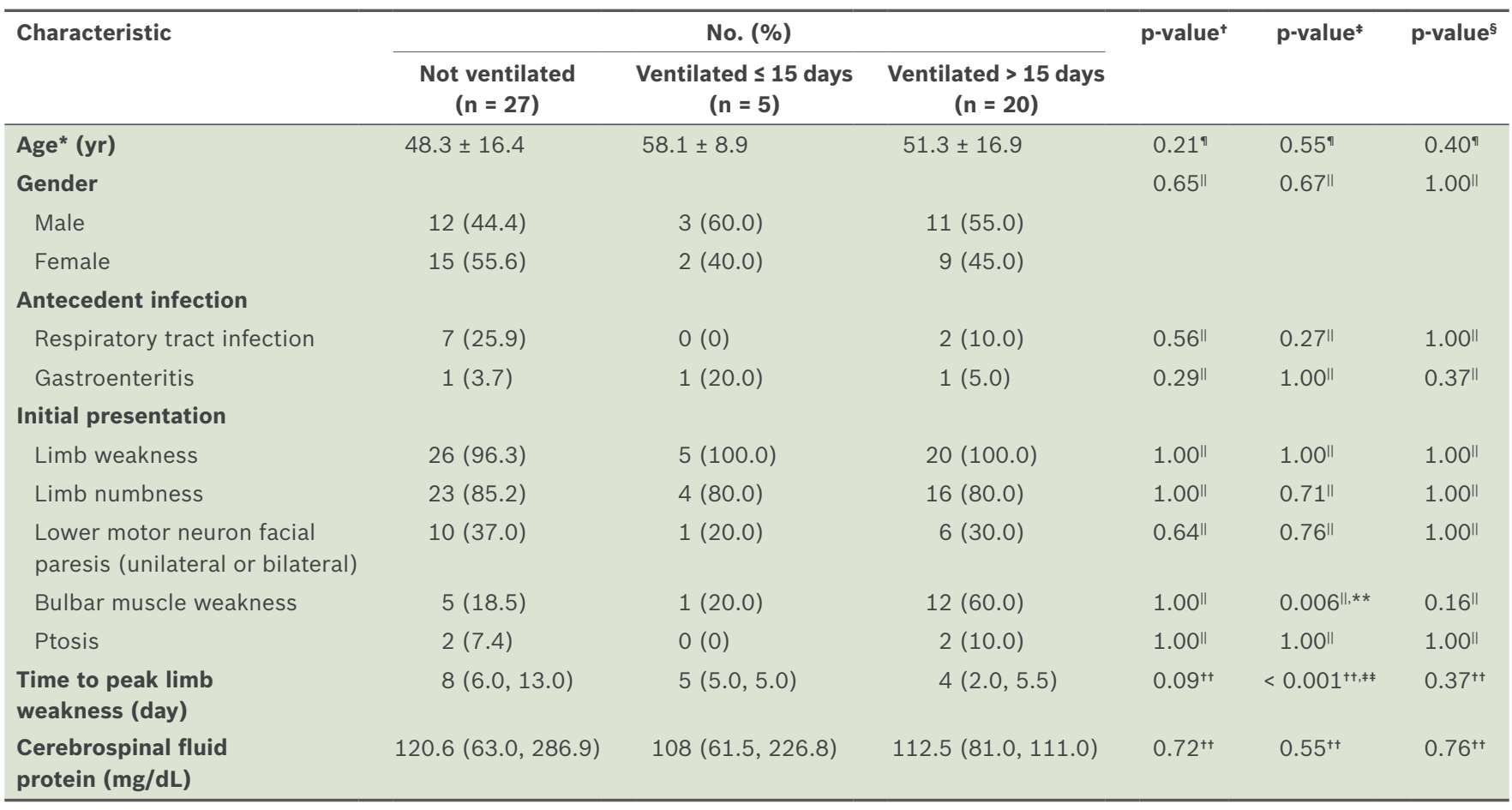

Note: Data for three patients who required mechanical ventilation was not available as the patients were discharged against advice or transferred to another institution. * Data presented as mean \pm standard deviation. †Comparison between patients who were not ventilated and patients who were ventilated for $\leq 15$ days. $¥$ Comparison between patients who were not ventilated and patients who were ventilated for $>15$ days. $\S$ Comparison between patients who were ventilated for $\leq 15$ days and

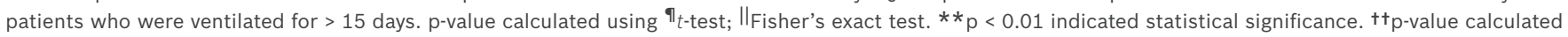
using Mann-Whitney $U$ test. ${ }^{\ddagger} \neq p<0.001$ indicated statistical significance.

\section{DISCUSSION}

In the present study, we found that the incidences of respiratory failure and requirement for prolonged mechanical ventilation among patients with GBS were higher in our institution than in others. Studies on GBS patients conducted in other tertiary care and medical teaching hospitals have found that the incidence of mechanical ventilation requirement ranged from $8 \%$ to $27 \%$. $^{(5,6)}$ The incidence of mechanical ventilation requirement in the present study was also higher than that reported in an international study (50.9\% vs. $25 \%) .{ }^{(7)}$ This stark contrast may be due to the smaller sample size that was used in the present study, as well as the fact that the present study was based on cases in a single hospital (i.e. there was the possibility of inclusion bias). As our institution is known to be a supertertiary medical care centre in Thailand, most severe cases of GBS would be transferred here for intensive treatment. Hence, nearly three-quarters (i.e. $72.7 \%$ ) of the patients admitted to our institution for GBS were bedridden and had respiratory insufficiency (HSS grades 4-5) on presentation.

The mean age of the GBS patients in the present study is comparable to that of previous reports. ${ }^{(5,6,8)}$ Although antecedent upper respiratory tract infections and gastroenteritis were commonly observed among GBS patients in previous studies $(50 \%){ }^{(5,6,8,9)}$ only $14(25.5 \%)$ of the patients in the present study were reported to have such infections.

Bulbar muscle weakness at presentation and time to peak limb weakness $\leq 5$ days were found to be significantly associated with the need for mechanical ventilation and its prolonged use
(> 15 days) in the present study. As our institution provides treatment to a greater proportion of patients with severe GBS, it is not uncommon that once mechanical ventilation support is initiated, it would be needed for a longer duration. In contrast, age, gender, ptosis, facial paresis, limb weakness, CSF white blood cell count, elevated CSF protein and treatment option showed no association with the need for mechanical ventilation or its prolonged use (> 15 days).

Various clinical presentations have been reported to be predictors of the need for mechanical ventilation. These include: advanced age; rapid disease progression; time from onset to admission of $<7$ days; inability to stand, cough or lift elbows or head; cranial nerve palsy; high HSS; dysautonomia; conduction block in electrophysiological study; facial or bulbar weakness; low Medical Research Council sum score; and anti-GQ1b antibody. ${ }^{(10-15)}$ One study, which investigated the requirement for prolonged mechanical ventilation among patients with GBS who were admitted to an intensive care unit, showed that an inability to perform foot flexion in association with a motor conduction block of the sciatic nerve after completing immunotherapy (either intravenous immunoglobulin or plasma exchange) predicted the need for prolonged mechanical ventilation (> 15 days). ${ }^{(8)}$

We believe that it is essential to identify the clinical presentations that can predict the need for longer mechanical ventilation support at admission, in order to enable timely and appropriate respiratory care for patients with GBS, especially if the hospital has limited advanced laboratory resources. Moreover, well-recognised significant predictors of severe 
respiratory insufficiency, such as abnormalities in CSF protein, electrophysiological results and the presence of antiganglioside antibodies, would not be apparent until the end of the second week of clinical onset. ${ }^{(11,16)}$ In other words, an overreliance on the results of these diagnostic tests may delay the identification of GBS patients with a high risk of severe respiratory disability. Furthermore, the occurrence of respiratory complications from prolonged intubation and artificial ventilation can be partly prevented by early tracheostomy. Tracheostomy should be initiated earlier in patients who have severe GBS and are at high risk for prolonged mechanical ventilation. Success in minimising ventilator-associated pulmonary infections will significantly improve the clinical outcome of patients with GBS.

The retrospective nature of the present study and the fact that it was conducted at a single tertiary medical centre limited the strength and generalisability of the study's results. Future systematic and prospective studies should be carried out to elucidate the overall nature of the respiratory insufficiency experienced by patients with GBS, as well as its associated complications.

\section{REFERENCES}

1. Guillain G, Barré JA, Strohl A. [Radioculoneuritis syndrome with hyperalbuminosis of cerebrospinal fluid without cellular reaction. Notes on clinical features and graphs of tendon reflexes. 1916]. Ann Med Interne (Paris) 1999; 150:24-32. French.

2. Vucic S, Kiernan MC, Cornblath DR. Guillain-Barré syndrome: an update. J Clin Neurosci 2009; 16:733-41.

3. McGrogan A, Madle GC, Seaman HE, de Vries CS. The epidemiology of Guillain-Barré syndrome worldwide. A systematic literature review. Neuroepidemiology 2009; 32:150-63.

4. Asbury AK, Cornblath DR. Assessment of current diagnostic criteria for Guillain-Barré syndrome. Ann Neurol 1990; 27 Suppl:S21-4.

5. Areeyapinan P, Phanthumchinda K. Guillain-Barre syndrome: a clinical study in King Chulalongkorn Memorial Hospital. J Med Assoc Thai 2010; 93:1150-5.

6. Padungkit N, Tiamkoa S. [The prognosis and predictors of Guillain-Barré Syndrome in Srinagarind Hospital]. North-Eastern Thai J Neurosci 2010; 5:24-33. Thai.

7. Hughes RA, Cornblath DR. Guillain-Barré syndrome. Lancet 2005; 366:1653-66.

8. Fourrier F, Robriquet L, Hurtevent JF, Spagnolo S. A simple functional marker to predict the need for prolonged mechanical ventilation in patients with Guillain-Barré syndrome. Crit Care 2011; 15:R65.

9. The prognosis and main prognostic indicators of Guillain-Barré syndrome. A multicentre prospective study of 297 patients. The Italian Guillain-Barré Study Group. Brain 1996; 119(Pt 6):2053-61.

10. Lawn ND, Fletcher DD, Henderson RD, Wolter TD, Wijdicks EF. Anticipating mechanical ventilation in Guillain-Barré syndrome. Arch Neurol 2001; 58:893-8.

11. Walgaard C, Lingsma HF, Ruts L, et al. Prediction of respiratory insufficiency in Guillain-Barré syndrome. Ann Neurol 2010; 67:781-7.

12. Durand MC, Porcher R, Orlikowski D, et al. Clinical and electrophysiological predictors of respiratory failure in Guillain-Barré syndrome: a prospective study. Lancet Neurol 2006; 5:1021-8.

13. Kaida K, Kusunoki S, Kanzaki M, et al. Anti-GQ1b antibody as a factor predictive of mechanical ventilation in Guillain-Barré syndrome. Neurology 2004; 62:821-4.

14. Funakoshi K, Kuwabara S, Odaka M, Hirata K, Yuki N. Clinical predictors of mechanical ventilation in Fisher/Guillain-Barré overlap syndrome. J Neurol Neurosurg Psychiatry 2009; 80:60-4.

15. Sharshar T, Chevret S, Bourdain F, Raphaël JC; French Cooperative Group on Plasma Exchange in Guillain-Barré Syndrome. Early predictors of mechanical ventilation in Guillain-Barré syndrome. Crit Care Med 2003; 31:278-83.

16. van Doorn PA, Ruts L, Jacobs BC. Clinical features, pathogenesis, and treatment of Guillain-Barré syndrome. Lancet Neurol 2008; 7:939-50. 